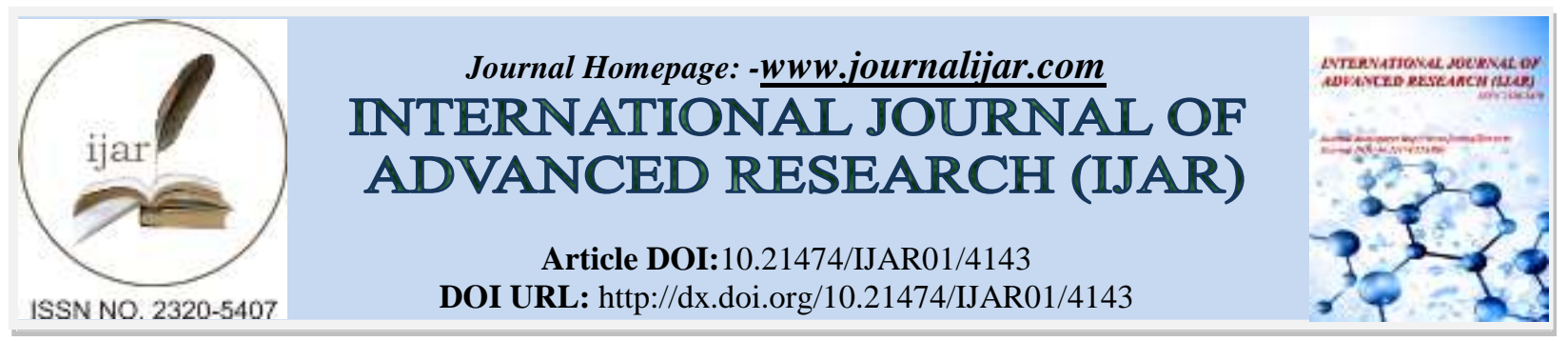

RESEARCH ARTICLE

\title{
A CASE STUDY OF AYURVEDIC MANAGEMENT ON SANDHIGAT VATA (UPASTAMBHIT) WITH STHAULYA (OBESITY).
}

\author{
Dr. Archana S. Dachewar ${ }^{1}$, Dr. Sneha P. Waghmare ${ }^{2}$ and Dr. Manjusha R. Dapurkar ${ }^{2}$. \\ 1. Professor and HOD kayachikitsa dept. \\ 2. PG Scholar, kayachikitsa dept.
}

\section{Manuscript Info}

Manuscript History

Received: 05 March 2017

Final Accepted: 06 April 2017

Published: May 2017

Key words:-

Sandhigat vata, sthaulya, janubasti, rasna guggul, triphala guggul, vishgarbha tail, aasana.

\section{Abstract}

Sandhigat vata is a disease of musculoskeletal system mainly affects the large joints. In modern medicine it is mainly correlated with inflammatory osteoarthritis. The main symptoms in this are pain in joints, difficulty in movements, swelling, tenderness and warmth over inflamed joints. Ayurveda included this disease under upastambhit sandhigat vata.

Sthaulya or obesity again contributes in symptoms and this again leads to increase the obesity. This vicious cycle hampers the patient's life dangerously. Here an effort was made to treat the patient with multiple ayurvedic treatment modalities. At the end of 30 days $90 \%$ improvement seen in patient.

Copy Right, IJAR, 2016,. All rights reserved.

\section{Introduction:-}

Sandhigat vata is of two types (dhatukshayajanya) and inflammatory (upastambhit). Here the patient is of upastambhit type. In this patient due to overeating or improper eating, kaphprakopa or aamdushti occur (inflammatory triggers ) which hampers the normal functions of vata and this saamvayu get situate in sandhipradesh and produces the symptoms like sandhigraha, sandhishotha, sandhivedana, etc. (inflammation of joints).

Sthaulya is also seen from above causes and due to obesity movements of joints especially lower limb joints and get affected and vicious cycle starts. Therefore here we were not concentrating on both the disease but the same cause for both the diseases and give treatment accordingly.

Aims And Objectives:-

A case study of Ayurvedic management on Sandhigat vata (upastambhit) with Sthaulya (obesity).

\section{Material and Methods:-}

Basic information of patient:-

Name- XYZ

Age- 67 yrs

Sex- male

Occupation- retired (previously LIC agent)

Socio economic status- middle class. 


\section{Chief complaints}

Rt- knee joint pain

Rt-knee joint swelling

Rt- knee joint redness and difficulty in moving

\section{History of present illness}

Three months before patient is suffering from mild pain in right knee joint. Subsequently the pain increases and patient suffers from swelling over it and difficulty during walking.

The stiffness increases to great extend since 15 Days .

\section{History of past illness}

No

4. Family History

No

\section{Treatment History}

No

6. Habitual History

History of over eating of rice, curd, sweet dishes, fermented food, milk maid dishes.

History of long distance walking during job

\section{Examination}

General examination - G.C. Mod

AEBE , R.R. $-20 / \mathrm{min}$

$\mathrm{S}_{1} \mathrm{~S}_{2-} \mathrm{N}, \mathrm{P}-84 / \mathrm{min}$

$\mathrm{P} / \mathrm{A}$ - Soft, Non tender

Liver Spleen - Non palpable

Local Examination - ( B/L knee joint examination $)$

\begin{tabular}{|c|c|c|}
\hline Sign & Left knee & Right knee \\
\hline 1. Rubor & - & ++ \\
\hline $2 . \quad$ Calor & - & ++ \\
\hline 3. Dolar & - & ++ \\
\hline 4. Tumor & - & ++ \\
\hline 5. Girth & & $163 \mathrm{~cm}$ \\
\hline Above Knee Jont $(5 \mathrm{~cm})$ & $160 \mathrm{~cm}$ & $158 \mathrm{~cm}$ \\
\hline$\quad$ At knee joint & $157 \mathrm{~cm}$ & $169 \mathrm{~cm}$ \\
\hline
\end{tabular}

8. Investigation

R.A factor.- Negative

ESR- $30 \mathrm{~mm} / \mathrm{hr}$

CPR-Negative

CBC-Under Normal limits

$\mathrm{X}$-ray Rt knee joint - AP, Lat- Osteophytes present.

\begin{tabular}{|l|l|l|l|}
\hline Nirupastambhit Sandhigat Vata & Aamvata & Vatarakta & Krostukshirsha \\
\hline
\end{tabular}




\begin{tabular}{|l|l|l|l|}
\hline Starts from big joint & $\begin{array}{l}\text { Starts from big and } \\
\text { small joint }\end{array}$ & Starts from small joint & Only in knee joint \\
\hline Pain during movements & $\begin{array}{l}\text { Pain relieves after } \\
\text { movement }\end{array}$ & - & - \\
\hline No Morning stiffness & $\begin{array}{l}\text { Morning stiffness } \\
\text { present for more than } \\
30 \text { min }\end{array}$ & No morning stiffness & No morning stiffness \\
\hline Local inflammatory signs absent & $\begin{array}{l}\text { Local inflammatory } \\
\text { signs present }\end{array}$ & $\begin{array}{l}\text { Local inflammatory } \\
\text { signs present }\end{array}$ & $\begin{array}{l}\text { Local inflammatory } \\
\text { signs present }\end{array}$ \\
\hline Fleeting movement absent & $\begin{array}{l}\text { Fleeting movement } \\
\text { present }\end{array}$ & $\begin{array}{l}\text { Fleeting movement } \\
\text { absent }\end{array}$ & $\begin{array}{l}\text { Fleeting movement } \\
\text { absent }\end{array}$ \\
\hline
\end{tabular}

9. Treatment protocol-

Total duration- 30 days

1. $1^{\text {st }} 8$ days- valuka pottali sweda

2. Next 8 days- vishagarbha tail for LA and valuka pottali sweda.

3. Next 14 days - janubasti with vishagarbha tail and valuka pottali sweda.

Internal medicine-( for 30 days)

1. Sunthi erand siddha lukewarm water to drink.

2. Rasna guggul 2 tab TDS.

3. Triphala guggul 2 tab BD.

4. Chitrakadi vati 2 tab BD.

5. Gandharva haritaki 2 tab BD.

6. Triphala churna kashaya $50 \mathrm{ml}+$ errand sneha $10 \mathrm{ml} \mathrm{HS}$.

Supportive medicine-

Aasana (yoga) - basic movements of limbs.

Hastapadasana.

\section{Diet plan}

-Avoid heavy food, sweet, milk made products, curd, rice, fermented food, stale food.

-Drink lukewarm water.

-Avoid heavy oil massage.

\section{Observation:-}

Assessment of patient for 30 days with ayurvedic treatment.

\begin{tabular}{|c|c|c|c|}
\hline signs & Day 1 & Day 15 & Day 30 \\
\hline & Rt. Knee joint & Rt. Knee joint & Rt. Knee joint \\
\hline 1.Rubor & ++ & + & - \\
\hline 2.Calor & ++ & + & $+/-$ \\
\hline 3.Dolar & ++ & ++ & - \\
\hline 4.Tumor & ++ & $163 \mathrm{~cm}$ & $160 \mathrm{~cm}$ \\
\hline 5.Girth & $163 \mathrm{~cm}$ & $157 \mathrm{~cm}$ & $157 \mathrm{~cm}$ \\
\hline Above Knee Jont ( \\
5 cm )
\end{tabular}

\section{Discussion:-}


The patient is observed for 30 days with above ayurvedic treatment, there is gradual decrease seen in inflammatory signs such as local redness ( rubor) decreases after 30 days, local temperature decreases after 30 days swelling reduces gradually within 30 days. Patient can walk without pain after treatment and is now able to do his day to day activities without anyone's help. This all seen because above treatment relieves vataprakopa and localized aam due to with patient fees pain in rt. knee joint.

\section{Conclusion:-}

After clinical assessment for 30 days with internal medicine, panchakarma and supportive treatment patient get relief about $90 \%$ and now patient is able to do his regular activities.

\section{References:-}

1. Acharya JT, editor. Reprint ed. Varanasi: Chaukhambha Prakashan; 2009. Charaka Samhita of Agnivesha, Sutra Sthana, Ch.25, Ver. 40; p. 132.

2. Golwalla AF, Golwalla SA. 21st ed. Mumbai: Asia Publishing House; 1970. Medicine for Students; p. 1032.

3. Ch. 321. 15th Edition 1987. Harrison's Principles of Internal Medicine.

4. Acharya Vaidya Jadavaji Trikamji., editor. Chaukhabha Sanskrita Sansthan. 37. Vol. 28. Varanasi: Chikitsasthan; Agnivesha, Charaka Samhita, with commentary Chakrapanidatta; p. 618.

5. Kumar \& Clark Clinical Medicine. 6th Edition. p. 551. Ch. 10.

6. Rose \& Wilson, Anatomy \& Physiology in Health \& Illness-Elsevier Churchill Livingstone. Ch. 11. :274.

7. Vagbhata. Astanga Hridayam (Vidyotinitika). Tripathi BN, editor, 1 st ed. Varanasi: Caukhamba Sanskrit Pratishthan; 2011. Nidansthana, 15/14-15. p. 539

8. Senani GS, Singhal BS, editors, Association of Physicians of India, API text book of medicine. 6 th ed. Mumbai: The Association of Physicians of India; 1999. Section 12, Chapter 28, Lumbar spondylosis. p.831.

9. Sushruta. Sushruta Samhita. Sastri AD, editor. 1 st ed. Varanasi: Caukhamba Sanskrit Pratishthna; 2010. Chikitsa sthana, 4/ 8. p.35

10. Acharya JT. SusruthaSamhita with Nibandhasangraha commentary of Dalhana. 1st ed. ChikitsaSthana;NiruhakramaChikitsa: Chapter 38, Verse 83.Varanasi (India): Chaukambha Sanskrit Sansthan; 2013. p. 546. 5. Acharya JT. S 